\title{
Monoclonal immunofluorescence compared with silver stain for investigating Pneumocystis carinii pneumonia
}

\author{
J Midgley, P A Parsons, D C Shanson, O A N Husain, N Francis
}

\begin{abstract}
Two hundred and eighty two specimens from 220 patients positive for HIV with respiratory tract symptoms, or febrile illness, or both, were examined for the presence of Pneumocystis carinii. Specimens were either induced sputum samples or bronchoalveolar lavage fluids. To establish the optimal method for laboratory diagnosis a comparison was made of detection of the organism by use of monoclonal antibody and immunofluorescence with conventional silver staining methods. Three commercially available reagents for immunofluorescence were also compared. Immunofluorescence was significantly more sensitive than the silver stain and the best results for immunofluorescence were obtained using Northumbria Biologicals Ltd reagents.
\end{abstract}

Most patients with AIDS have at least one episode of Pneumocystis carinii pneumonia. Clinical management of these patients is assisted by continued rational treatment once a prompt diagnosis is established, followed by appropriate prophylaxis.

Improved methods for obtaining induced sputum and the use of monoclonal antibodies for immunofluorescence techniques have increased the confirmatory diagnosis rate of these infections. ${ }^{1-4}$ We have extended our study which compared the immunofluorescence method with a modified Grocott silver stain $^{6}$ and also compared three monoclonal antibodies which are available commercially in the United Kingdom from Northumbria Biologicals Ltd, Meridian Diagnostics Inc, and Dako Ltd.

Department of
Microbiology,
St Stephen's and
Westminster
Hospitals, Horseferry
Road, London
SW1P 2AR
J Midgley
D C Shanson
Department of
Cytology, Charing
Cross Hospital,
London
P A Parsons
O A N Husain
Department of
Histopathology,
Charing Cross and
Westminster Medical
Schools, London
N Francis
Accepted for publication
19 July 1990

Microbiology,

St Stephen's and

Westminster

Hospitals, Horseferry

SWIP 2AR

J Midgley

D C Shanson

Department of

Cytology, Charing

Hospital

Department of

Histopathology,

Charing Cross and

Westminster Medical

N Francis

19 July 1990

\section{Methods}

Between November 1988 and July 1989, 282 specimens from the respiratory tract of patients positive for human immunodeficiency virus (HIV) with suspected Pneumocystis pneumonia were investigated for the presence of Pneumocystis carinii. Methods for obtaining induced sputum specimens and bronchoalveolar lavage (BAL) fluids were as described by Leigh et al..$^{5}$ After a negative result for Pneumocystis on induced sputum the patient underwent bronchoscopy, if possible, within four days. Specimens were handled promptly in the laboratory; close cooperation between ward and laboratory was essential for efficient processing of this clinical material. Induced sputum samples were first homogenised with "Sputasol" for about 10 minutes. Occasionally BAL fluids were homogenised if the consistency was very viscous. Specimens at this stage were divided into several aliquots to investigate for other pathogens including Mycobacteria, Legionella, Cryptococcus, Candida and Aspergillus. The only viral pathogen sought was cytomegalovirus which was undertaken for us by the Virology Department at the Royal Free Hospital.

Both homogenised induced sputum and BAL specimens were centrifuged for $10 \mathrm{~min}$ utes at $2000 \mathrm{rpm}$, the supernatant was removed, and the cell pellets obtained were used to smear slides for staining by the modified Grocott technique, as described previously. ${ }^{5}$ The deposit was resuspended in sterile distilled water, washed twice by repeat centrifugation, and resuspended in distilled water, before using the cellular material to prepare slides for immunofluorescence. Procedures were carried out according to the manufacturer's recommendations, high quality acetone was used to fix the slides to be stained by Northumbria Biologicals (NBL) and Dako reagents, but heat fixing was recommended by Meridian. All the slides were treated with enzyme for 30 minutes before incubating with anti-P carinii antibody followed by conjugate.

The slides were then viewed under ultraviolet light. Where more than five cysts or clusters of cysts were seen a positive result was recorded; specimens showing one to five cysts were recorded as equivocal.

\section{Results}

Tables 1 and 2 give the results of the comparison of immunofluorescence with silver stain.

\section{Discussion}

No specimens were positive with silver staining and negative by immunofluorescence, as has been reported in the USA. ${ }^{2}$ There were two occasions, however, where a possible error in technique was indicated by the presence of cysts stained by silver alone, but these could clearly be seen by immunofluorescence when repeated. We do not therefore advocate the use of immunofluorescence alone. Monoclonal antibodies have been reported not to react with cysts from every case encountered, ${ }^{7}$ and some manufacturers state that their monoclonal antibody will react with trophozoites as well as with cyst forms. Non-immunological methods 
Table 1 Investigations with similar results for immunofluorescence and silver staining methods

\begin{tabular}{llll}
\hline & Immunofluorescence & Silver & No of patients \\
\hline Induced sputum & Positive & Positive & 39 \\
BAL & Positive & Positive & $14^{\star}$ \\
Induced sputum and subsequent & & & \\
BAL & Negative & Negative & 52 \\
Induced sputum only & Negative & Negative & 76 \\
BAL only & Negative & Negative & 21 \\
\hline
\end{tabular}

*Six patients without induced sputum. Five patients with induced sputum negative by both techniques. Three patients with induced sputum positive by immunofluorescence alone.

Table 2 Investigations where descrepant results were obtained by immunofluorescence and silver staining methods

\begin{tabular}{|c|c|c|c|}
\hline & Immunofluorescence & Silver & No of patients \\
\hline $\begin{array}{l}\text { Induced sputum } \\
\text { Induced sputum and subsequent }\end{array}$ & Positive & Negative & $9 \star$ \\
\hline $\begin{array}{l}\text { BAL } \\
\text { BAL } \\
\text { Induced sputum }\end{array}$ & $\begin{array}{l}\text { Positive } \\
\text { Positive } \\
\text { Equivocal }\end{array}$ & $\begin{array}{l}\text { Negative } \\
\text { Negative } \\
\text { Negative }\end{array}$ & $\begin{array}{l}1 \\
3 \\
6 \dagger\end{array}$ \\
\hline $\begin{array}{l}\text { Induced sputum and subsequent } \\
\text { BAL } \\
\text { BAL }\end{array}$ & $\begin{array}{l}\text { Equivocal } \\
\text { Equivocal }\end{array}$ & $\begin{array}{l}\text { Negative } \\
\text { Negative }\end{array}$ & 1 \\
\hline
\end{tabular}

$\star$ Three patients with subsequent BAL positive by both techniques (table 2). Five patients with no subsequent BAL. One patient with a previous episode of $P$ carinii infection one month earlier.

$\uparrow$ Two patients with subsequent BAL positive by both techniques; one patient with subsequent TWo patients with subsequent BAL positive by both techniques; one patient with subsequent
BAL positive by immunofluorescence alone; one patient with no subsequent BAL; one patient with no subsequent BAL but necropsy lung tissue positive by immunofluorescence; one patien with no subsequent BAL but further induced sputum one month later positive by both techniques.

Table 3 Comparison of three commercially available immunofluorescence reagents using induced sputum samples from patients with suspected Pneumocystis infection

\begin{tabular}{lllll}
\hline & NBL & Meridian & Dako & No of patients \\
\hline & Positive & Positive & Positive & 4 \\
& Positive & Equivocal & Equivocal & 3 \\
& Positive & Negative & Negative & 3 \\
Total clearly positive & Equivocal & Negative & Negative & 2 \\
\hline
\end{tabular}

methods and newer monoclonal antibodies will continue to be included in our studies.

Positive immunofluorescence with a typical arrangement of clusters of cysts was similar with all three immunofluorescence reagents when large numbers of cysts - that is, greater than 20-were present, and this was found in only four of the 12 patients investigated by all three methods (table 3 ). Meridian state that the presence of two or more cysts should be considered positive for $P$ carinii. We were not able to report confidently on such low numbers on any of the specimens stained by either Meridian or Dako. In neither of these methods was a counterstain used. With NBL reagents, however, low numbers of cysts, even in the absence of clusters, were distinctly visible under both low and high power magnification.
These reagents include the counterstain Evans Blue which may facilitate the recognition of single cysts from background material. The NBL reagents are available in kit form with full instructions, providing a technique which can be easily used by any laboratory with access to fluorescence microscopy. The remainder of our specimens were stained by NBL immunofluorescence reagents and our modified Grocott method only.

We found the immunofluorescence technique to be reliable, repeatable, easy to carry out, and to give well defined cyst fluorescence with minimal background staining. The method of preparation for the immunofluorescence method is straightforward with timed incubations. In contrast, the Grocott method is unreliable, needing considerable technical skill to judge when the slide is stained adequately. Over- or understaining may make a slide unreadable. Preparation time is less for the Grocott method as we speed up the incubation time by heating in a microwave oven, but screening time is considerably more than for the immunofluorescence stained slides. Care is needed to avoid confusion with yeasts which are often present in these specimens. The presence of $P$ carinii could be shown in specimens from 63 patients in this study, 10 of these by immunofluorescence alone. In a further three patients the possibility of the presence of $P$ carinii was indicated by equivocal immunofluorescence. Our results indicate an increase in sensitivity for the detection of $P$ carinii in cytological preparations of induced sputum using the immunofluorescence technique.

We are grateful to our clinical colleagues at St Stephen's Clinic and the Westminster Hospital, including Drs Collins, Gazzard, Leigh and Smith, for providing the respiratory samples used in this laboratory study.

1 Kovacs JA, Gill VJ, Swan JC, et al. Prospective evaluation of a monoclonal antibody in diagnosis of Pneumocystis carinii pneumonia. Lancet 1986;ii:1-3.

2 Gill VJ, Evans G, Stock F, et al. Detection of Pneumocystis carinii by fluorescent antibody stain using a combination
of three monoclonal antibodies. J Clin Microbiol 1987; of three monocl

3 Dournon E, Rajagopalan P, Albert F, et al. Diagnosis of Pneumocystis carinii pneumonia by non-experts. Lancet 1989;i:107.

4 Kovacs JA, Ng VL, Masur $\mathrm{H}$, et al. Diagnosis of Pneumocystis carinii pneumonia: Improved detection in sputum with use of monoclonal antibodies. N Engl J Med 1988;318:589-93.

5 Leigh TR, Parsons P, Hume C, Husain OAN, Gazzard B, Collins JV. Induced sputum for diagnosis of Pneumocystis carinii pneumonia. Lancet 1989;ii:205-6.

6 Midgley J, Parsons PA, Shanson DC, et al. Increased sensitivity of Immunofluorescence for detection of sensitivity of Immunofluorescence for

7 Kovacs JA, Halpen JL, Lundgren B, et al. Monoclonal antibodies to Pneumocystis carinii: Identification of specific antigens and characterization of antigenic differences between rat and human isolates. $J$ Infect Dis 1989;159:60-70. 